\title{
Automatic Semantic Content Extraction from Videos using Genetic Algorithm
}

\author{
Sruthi Rose Boban \\ PG Student, \\ Department of Computer \\ Science and Engineering, \\ Mar Athanasius College of \\ Engineering, \\ Kothamangalam, Kerala, India
}

\author{
Aby Abahai .T \\ Assistant. professor, \\ Department of Computer \\ Science and Engineering \\ Mar Athanasius college of \\ engineering \\ Kothamangalam, Kerala, \\ India.
}

\author{
Linda Sara Mathew \\ Assistant. professor \\ Department of Computer \\ Science and Engineering \\ Mar Athanasius college of \\ engineering \\ Kothamangalam, Kerala, \\ India.
}

\begin{abstract}
Motivated by the needs of semantic search and retrieval of multimedia contents, operating directly on the video based annotations can be thought as a reasonable way for meeting these needs as video is a common standard providing a wide multimedia content description schema. Raw data and lowlevel features alone are not satisfactory to fulfil the user's requirements; that means, a deeper understanding of the content at the semantic level is necessary. A semantic content extraction system that allows the user to query and regain objects, events, and concepts that are extracted automatically is proposed. In automatic extraction process, starts with object and define class for each process in video data. Objects extracted from consecutive representative frames are processed to extract temporal relations. In addition to that, additional rule to lower spatial relation computation cost and to be able to define some difficult situations more successfully is used. Event extraction process uses objects. Similarly, objects and events are used in concept extraction process.
\end{abstract}

\section{Keywords}

Content-based retrieval, fuzziness, ontology, Semantic content extraction, video content modeling.

\section{INTRODUCTION}

Video contents collected from video data is the main building block elements of the infrastructure. Video data exist for educational the video data and it is generated manually using an explanation tool instead of the automatic extraction techniques such as text mining, video processing,.. This is skilled through the development of an ontology-based semantic content model with that semantic content extraction algorithms. Here work differs from other semantic content extraction and representation studies in lots of ways and contributes to semantic video modeling and semantic content extractions investigate areas. First of all, a metaontology, rule creation standard which is area autonomous, to create domain ontologies. Domain ontologies are including extra law definitions. The achievement of the automatic semantic content extraction framework is fuzziness in class and relation definitions in the model and in rule definitions.

As a consequence of up to date advances of World Wide Web, dramatic increases in the sum of multimedia data have revealed the needs for the semantic retrieval techniques of multimedia contents as the high amount of multimedia data needs high level of services.User requests about gaining the ability of querying the individual multimedia data semantically play an important role on the researches in the field. The need mainly results from the personalization needs of the users. In other words, the complexity of computer users' management needs on multimedia contents causes them to direct themselves into a way in which the multimedia contents are managed according to the personal interest areas. Clearly, incorporating prior knowledge is a prerequisite for the reclamation as well, to permit appreciative of the semantics of user investigate, and subsequently, aligning and same with the content explanation semantics. Proprietary information representation solutions, while efficient inside the predefined usage situation, impose grave boundaries considering reusability and interoperability. Underpinned with the appearance of the Semantic Web, the need for shareable, suitably rich, understanding demonstration formalisms has revived interest and gave rise to advanced knowledge modelling solutions. Ontologies, promising a common and shared accepting of a domain, have twisted up into explanation enabling technologies providing machine reasonable semantics. Furthermore, due to the specific semantics, ontologies provide automated deduction maintain that can further enhance retrieval by exploiting hidden conceptual associations discovery and derivation of new ones for realizing semantic-based recommendation services. Consequently, next the ontology-based concept for the illustration of the explanation metadata entails major compensation for the reason of semantic explore and retrieval. More in particular, dissimilar keyword-based explanation, ontology-based explanation do not rely on plain word similar, and thus administer to defeat the syntactic boundaries that keyword-based investigate and retrieval systems. Synonyms and homonyms, two general reasons accounting for little recall and accuracy respectively in established keyword-based approaches do not have any proposition when content images are planned based on ontologies Ontology provides many reward and capabilities for content modelling. Yet, a huge popular of the ontology based video content modelling studies offer domain definite ontology models restrictive its use to a detailed domain. Besides, generic ontology models make available solutions for multimedia formation representations. In this study, offer a wide-domain applicable video content model in command to model the semantic content in videos. Therefore, using semantic entities in the explanation helps us as an upgrading for the data model. "Objects" and "Events" are the primitives used to construction the explanation for semantics. Objects are the entities recounting the concepts while measures are used to model the relations between the concepts and relating the performance in the video segments. Therefore, the querying performance thorough the videos are processed using the analysis actions working on the ontology instances of each video represented by SWRL. In previous language, the answers for the queries on the video contents 
are extracted by analysis on the logical illustration. The infrastructure proposed in this thesis uses a video data model based on explanation layering. But, an object-based move towards is also used in the explanation in order to hybridize this data model with the object-based models for structuring the video explanation.

\section{RELATED WORKS}

Recent solutions are silent far from success the ultimate objective, namely to facilitate users to retrieve the required video clip among huge amounts of visual data in a semantically important manner. Objects and/or events use all these information to make events and concepts. In [5], the organization has a consistent video data model, which gives the client the aptitude to create ontology-supported fuzzy querying. RDF is used to characterize metadata. OWL is used to correspond to ontology and RDQL is used for querying. Queries contain items, events, spatio-temporal clauses; concepts and low-level character are handled. A video database model (OVDAM) provides automatic object, event and concept extraction. With using instruction sets and authority opinions, low-level attribute values for objects and associations between items are determined. The genetic algorithm-based classifier is old to formulate organization of segments to objects. At the apex level ontology of objects, events and concepts are worn. Multimedia databases have gained attractiveness due to quickly growing quantities of multimedia data and the have to to achieve efficient indexing, retrieval and investigation of this data. One consequence of multimedia databases is the requirement to development the data for feature extraction and labelling earlier to storage and querying. Huge quantity of data makes it unfeasible to entire this task yourself. Aims to decrease the effort for physical collection and group of objects appreciably by detecting and tracking the most important objects, and hence, requiring to come in the label for every object only formerly within each shot as a substitute of specifying the labels for every object in each frame they show. This is also necessary as a first step in a fully-automatic video database management system in which the labelling should also be completed automatically. Planned framework covers a scalable construction for video dispensation and stages of shot limit detection, most important object detection and tracking, and knowledge-based construction for successful spatio-temporal object querying in [10].

Multi-modal structure for semantic event extraction from basketball games based on webcasting text and broadcast video. Event recognition in sports video which relies heavily on low-level features nonstop extracted from video itself, our move towards aims to suspension bridge the semantic gap between low-level features and high-level events and facilitates personalization of the sports video. Promising grades in [3] are reported on real-world video clips by using text analysis, video analysis and text/video configuration. An unsupervised clustering based system as a substitute of predefined keywords to automatically detect event from webcasting text; get better the game time recognition algorithm to identify the break time in basketball games; a numerical approach as a substitute of finite state machine as in to detect event boundary in the video.

\section{SYSTEM MODEL}

\subsection{Overview}

VISCOM is a well-defined metaontology for construct area ontologies and acts as an substitute to the rule-based and domain-dependent removal methods. It contains classes and associations between these module. various of the module characterize semantic content types such as item and incident though others are used in the automatic semantic content extraction method. Associations defined in VISCOM provide ability to model actions and concepts associated with other items and actions.

Ontology provides many advantages and capabilities for content modeling. Yet, a great majority of the ontology based video content modeling studies propose domain specific ontology models limiting its use to a specific domain. Besides, generic ontology models provide solutions for multimedia structure representations. A wide-domain applicable video content model in order to model the semantic content in videos. In addition to the complexity of handling such difference, each rule structure can have weaknesses. Besides, VISCOM provides a standardized rule construction ability with the help of its metaontology. It eases the rule construction process and makes its use on larger video data possible. The rules that can be constructed via VISCOM ontology can cover most of the event definitions for a wide variety of domains. However, there can be some exceptional situations that the ontology definitions cannot cover. To handle such cases, VISCOM provides an additional rule based modeling capability without using ontology.

Hence, VISCOM provides a solution that is applicable on a wide variety of domain videos. Objects, events, concepts, spatial and temporal relations are components of this generic ontology-based model.

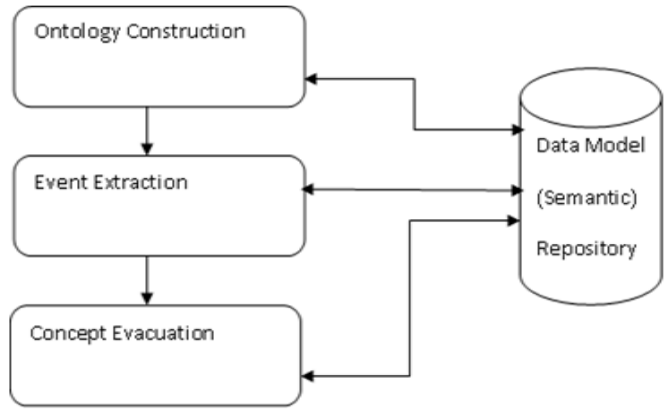

Fig 1. Architecture

\subsection{Ontology-Based Modeling}

VISCOM contains module and associations among these module. various of the module characterize semantic content types such as item and occasion while others are worn in the automatic semantic pleased extraction process. VISCOM module, VISCOM facts Properties correlate module with constants and VISCOM Object Properties are worn to describe associations. The linguistic part of VISCOM contains classes and relations between these classes.

Some of the classes represent semantic content types such as Object and Event while others are used in the automatic semantic content extraction process. Relations defined in VISCOM give ability to model events and concepts related with other objects and events. VISCOM is developed on an ontology-based structure where semantic content types and relations between these types are collected under VISCOM Classes, VISCOM Data properties which associate classes with constants and VISCOM Object Properties which are used to define relations between classes. 


\subsection{Domain Automatic Semantic Content Extraction Framework}

There are two main steps follow in the automatic semantic content extraction process. The following step is to take out and classify object instances beginning delegate frame of shots of the video instances. The third step is to extract measures and concepts by with area ontology and law definitions. A set of actions is executed to extract semantically important mechanism in the automatic event and concept extraction method. The first semantically important components are spatial relation instances among object instances. Then, the temporal associations are extracted by with changes in spatial relations. Lastly, actions and concepts are extracted by resources of the spatial and temporal relations.

\subsection{Object Extraction}

Object extraction techniques use instruction data to study object definitions, which are regularly figure, shade, and texture features. These definitions are frequently the similar across dissimilar domains. Through the object extraction procedure, for each commissioner key structure in the video, aforementioned object extraction method is performed and a position of objects is extracted and confidential. The extracted item instances are stored with their kind, frame digit, association value, and least Bounding quadrilateral data.

The basic idea is that local object appearance and shape can often be characterized rather well by the distribution of local intensity gradients or edge directions, even without precise knowledge of the corresponding gradient or edge positions. In practice this is implemented by dividing the image window into small spatial regions (.cells.), for each cell accumulating a local 1-D histogram of gradient directions or edge orientations over the pixels of the cell. The combined histogram entries form the representation. For better invariance to illumination, shadowing, etc., it is also useful to contrast-normalize the local responses before using them. This can be done by accumulating a measure of local histogram energy. over somewhat larger spatial regions (.blocks.) and using the results to normalize all of the cells in the block. It will refer to the normalized descriptor blocks as Histogram of Oriented Gradient (HOG) descriptors. Tiling the detection window with a dense (in fact, overlapping) grid of HOG descriptors and using the combined feature vector in a conventional SVM based window classifier gives our human detection chain. The detector window is tiled with a grid of overlapping blocks in which Histogram of Oriented Gradient feature vectors are extracted. The combined vectors are fed to a linear SVM for object/non-object classification.

\subsection{Event Extraction}

Event instances are extracted behind a series of automatic extraction processes. Every extraction method outputs instances of a semantic content form definite as an character in the domain ontology. Through the extraction process, the semantic substance is extracted among a confidence degree between 0 and 1 . An extracted event instance is represent among a type, a frame set instead of the event's gap, a connection value and the roles of the items enchanting part in the event. outline Set is used to represent the frame interval of instances. 6. Concept Extraction In the model extraction process, Concept Component persons and extracted object, event, and concept instances are worn. Concept Component persons recount objects, events, and concepts with concepts. When an object or event that is worn in the meaning of a conception is extracted, the linked concept instance is automatically extracted with the significance degree given in its definition. In addition, connection individuals are utilized in classify to extract added concepts from the extracted apparatus. The last step in the concept extraction process is executing model rule definition.

\subsection{Domain Ontology Construction}

Domain ontology was residential to confine the examined domain semantics and offer the conceptualization and terms for the explanation is shown in Fig 2. While through conceptbased browsing, question formulation is extensively facilitated. Seen from the examination perception, domain concepts detection is assisted by the information provided in the domain ontology definition, while extensibility and interoperability is difficult as all examination aspect are treated as ontological concepts. For all measures in process, do whether each occurrence has a different event inside it or not. If it has an occurrence then classify event in conditions of experience definition. Also check weather event define with other event temporal relative, then define event in terms of event temporal relative.

\subsection{VISCOM}

VISCOM is residential on an ontology-based construction where semantic content types and dealings between these types are together below VISCOM module, VISCOM information Properties which associate classes with constants and VISCOM article Properties which are used to describe dealings between module. In Accumulation, there are several domain autonomous class persons. The VISCOM properties consist of four extra relative like temporal relation, item relation, association persons and spatial relative. These relations are opening out by group, since these relative are distinct by classes itself where the predicate persons is used to denote "an entity is distinct as an personality of a class" in the formal demonstration of classes. It is sub-classed to the dissimilar types of domain concepts that must to be supported by the examination.

\subsection{Concept Extraction}

Concept is extract from information model is shown in Fig.2. Concepts recognition is assisted by the information provided in the domain ontology definition, while extensibility and interoperability is superior as all examination aspects are treat as ontological concepts. In the concept extraction practice, Concept section persons and extracted item, event, and perception instances are used. idea module individuals narrate objects, events, and concepts with concepts. When an item or occasion that is used in the meaning of a concept is extracted, the linked concept instance is automatically extracted with the significance degree specified in its definition. In addition, connection persons are utilized in direct to extract more concepts from the extracted mechanism. The last step in the concept extraction method is executing notion rule definition. Related to the event extraction, concepts are extracted with a partisanship value between 0 and 1 . 


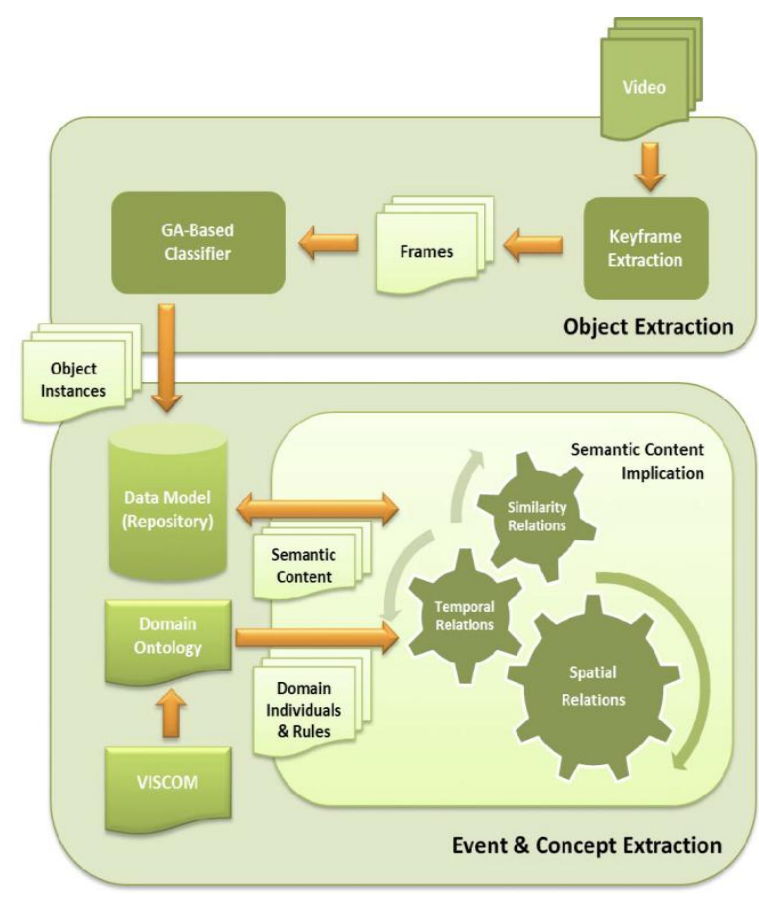

Fig 2. Automatic semantic content extraction framework.

\section{CONCLUSIONS}

The semantic content extraction procedure is completed mechanically. In adding, a general ontology-based semantic meta ontology representation for videos (VISCOM) is projected. Furthermore, the semantic content symbol potential and extraction achievement are enhanced by accumulation fuzziness in class, relative, and rule definitions. An automatic heritable Algorithm-based item extraction method is included to the planned system to imprison semantic content. In every module of the structure, ontology-based modeling and extraction capability are used. Object or event that be used in the explanation of a thought is extracted; the associated concept instance is repeatedly extracted with the application degree specified in its designation.

\section{REFERENCES}

[1] T. Yilmaz, Object Extraction from Images/Videos Using a Genetic Algorithm Based Approach, master's thesis, Computer Eng. Dept., METU, Turkey

[2] C. Xu, J. Wang, K. Wan, Y. Li, and L. Duan, Live Sports Event Detection Based on Broadcast Video and WebCasting Text, MULTIMEDIA '06: Proc. 14th Ann. ACM Int'l Conf. Multimedia, pp. 221-230

[3] Y. Zhang, C. Xu, Y. Rui, J. Wang, and H. Lu, Semantic Event Extraction from Basketball Games Using MultiModal Analysis, Proc. IEEE Int'l Conf. Multimedia and Expo (ICME '07), pp. 2190- 2193

[4] D. Song, H.T. Liu, M. Cho, H. Kim, and P. Kim, Domain Knowledge Ontology Building for Semantic Video Event Description, Proc. Int'l Conf. Image and Video Retrieval (CIVR), pp. 267-275

[5] Y. Yildirim, T. Yilmaz, and A. Yazici, OntologySupported Object and Event Extraction with a Genetic Algorithms Approach for Object Classification, Proc. Sixth ACM Int'l Conf. Image and Video Retrieval (CIVR '07), pp. 202-209

[6] Y. Yildirim and A. Yazici, Ontology-Supported Video Modeling and Retrieval, Proc. Fourth Int'l Conf. Adaptive Multimedia Retrieval: User, Context, and Feedback (AMR), pp. 28- 41

[7] Y. Yildirim, Automatic Semantic Content Extraction in Video Using a Spatio-Temporal Ontology Model, PhD dissertation, Computer Eng. Dept., METU, Turkey

[8] A.D. Bagdanov, M. Bertini, A. Del Bimbo, C. Torniai, and G. Serra, Semantic Annotation and Retrieval of Video Events Using Multimedia Ontologies, Proc. IEEE Int'l Conf. Semantic Computing (ICSC)

[9] L. Bai, S.Y. Lao, G. Jones, and A.F. Smeaton, Video Semantic Content Analysis Based on Ontology, IMVIP '07: Proc. 11th Int'l Machine Vision and Image Processing Conf.

[10] T. Sevilmis M. Bastan, U. Gu“du“ kbay, and O “. Ulusoy, Automatic Detection of Salient Objects and Spatial Relations in Videos for a Video Database System, Image Vision Computing, vol. 26, no. 10, pp. 1384-1396 